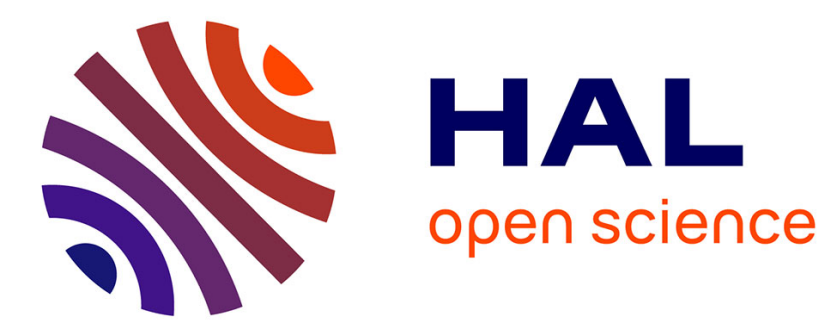

\title{
La ponctuation : problématiques linguistiques
}

\author{
Julien Rault
}

\section{To cite this version:}

Julien Rault. La ponctuation : problématiques linguistiques. Le Français Aujourd'hui, 2014, n 187 , p. 9-18. 10.3917/lfa.187.0009 . hal-01644414

\section{HAL Id: hal-01644414 https://hal.science/hal-01644414}

Submitted on 29 Nov 2017

HAL is a multi-disciplinary open access archive for the deposit and dissemination of scientific research documents, whether they are published or not. The documents may come from teaching and research institutions in France or abroad, or from public or private research centers.
L'archive ouverte pluridisciplinaire HAL, est destinée au dépôt et à la diffusion de documents scientifiques de niveau recherche, publiés ou non, émanant des établissements d'enseignement et de recherche français ou étrangers, des laboratoires publics ou privés. 


\title{
LA PONCTUATION : PROBLÉMATIQUES LINGUISTIQUES Julien Rault
}

\author{
Armand Colin | « Le français aujourd'hui »
}

$2014 / 4 n^{\circ} 187 \mid$ pages 9 à 18

ISSN 0184-7732

ISBN 9782200929459

Article disponible en ligne à l'adresse :

https://www.cairn.info/revue-le-francais-aujourd-hui-2014-4-page-9.htm

\section{Pour citer cet article :}

Julien Rault, «La ponctuation : problématiques linguistiques 》, Le français aujourd'hui 2014/4 ( $\left.\mathrm{n}^{\circ} 187\right)$, p. 9-18.

DOI 10.3917/lfa.187.0009

Distribution électronique Cairn.info pour Armand Colin.

(C) Armand Colin. Tous droits réservés pour tous pays.

La reproduction ou représentation de cet article, notamment par photocopie, n'est autorisée que dans les limites des conditions générales d'utilisation du site ou, le cas échéant, des conditions générales de la licence souscrite par votre établissement. Toute autre reproduction ou représentation, en tout ou partie, sous quelque forme et de quelque manière que ce soit, est interdite sauf accord préalable et écrit de l'éditeur, en dehors des cas prévus par la législation en vigueur en France. Il est précisé que son stockage dans une base de données est également interdit. 


\title{
LA PONCTUATION : PROBLÉMATIQUES LINGUISTIQUES
}

\author{
Julien RAULT \\ Université de Poitiers \\ Laboratoire FoReLL, B1
}

Comment vont évoluer ces signes artificiels insérés entre les mots et les phrases des textes imprimés ? Est-ce que ces signes artificiels resteront lettres mortes, obéissant à des règles arbitraires? Ou bien, une fois intégrés à la langue, vont-ils se transformer en lettres vives?

Yvan Fonagy, "Structure sémantique des signes de ponctuation », 1980.

L'étude de la ponctuation impose de se confronter à deux difficultés majeures : « donner une définition stable de la ponctuation, des signes et de leur fonction et choisir de les situer quelque part entre la transcription défaillante de l'oral, et le code graphique " (Pétillon 2002:13). Ces deux aspects, qui ont donné lieu à de nombreux travaux, sont intimement liés, l'approche définitoire étant nécessairement entravée par la référence à l'oral dont les critères pneumatiques (la voix, le souffle, le repos) s'accordent mal avec la réflexion linguistique; c'est bien en se dégageant progressivement du phonocentrisme qu'une véritable étude de la ponctuation a pu advenir, en posant notamment, depuis quelques années, les enjeux en termes sémiotiques.

Le discours sur la ponctuation a connu une évolution déterminante lors de la grande entreprise de normalisation des XVIII et $\mathrm{XIX}^{\mathrm{e}}$ siècles, laquelle, avançant une restriction des usages fondée sur l'établissement de critères syntaxiques et sémantiques, a entrainé un encodage grammatical. D’un point de vue épistémologique, le fait que le phonocentrisme ne soit plus la référence ultime des spécialistes de la langue a ouvert la voie à une approche grammaticale; les courants phonographique ${ }^{1}$ puis autonomiste ${ }^{2}$, au $\mathrm{XX}^{\mathrm{e}}$ siècle, interrogent dans ce contexte le rôle spécifique des signes de ponctuation dans le système de l'écrit.

1. Le courant phonographique se distingue du phonocentrisme en ce qu'il insiste sur les problèmes de corrélations entre l'intonation et les signes de ponctuation ; il considère que ces derniers participent à la fois à l'encodage de l'oral, mais servent aussi à marquer les relations syntaxiques (Catach 1980, 1994 ; Védénina 1989).

2. La conception autonomiste, qui fait de la langue écrite un système autonome, envisage de fait la ponctuation comme un ensemble de signes appartenant en propre à la forme écrite de la langue, sans lien nécessaire avec une dimension prosodique (Anis 1983, 2004). 
Ce phénomène particulier de grammaticalisation, au sens où une entité linguistique acquiert une fonction grammaticale, nous permet d'envisager un autre aspect majeur : l'instanciation d'un trait sémantique ou d'une valeur minimale. Cette spécialisation sémantique, implicite, au cœur du processus de grammaticalisation, est très certainement à l'origine du questionnement contemporain sur la nature sémiotique et, partant, sur la possibilité de considérer les signes de ponctuation comme de véritables signes linguistiques. De la grammaticalisation à la "structure sémantique » d'Y. Fonagy (1980) et au "signifié en langue » de J. Authier-Revuz (1995 : 136-137), se dessine un large mouvement d'inclusion dans le système de la langue, sous forme de sémiotisation ${ }^{3}$ : les "ponctuants", dont l'appréhension oscillait entre conception pneumatique et conception syntaxique, se sont progressivement grammaticalisés pour mieux se sémiotiser, et devenir de véritables signes de langue écrite.

\section{De l'oral à l'écrit}

\section{Usages et règles}

Les signes de ponctuation sont de moins en moins envisagés, dans le discours des spécialistes, comme des éléments prosodiques servant à transcrire l'intonation de l'oral. La mise au premier plan de leur rôle syntaxique et sémantique s'est progressivement généralisée dans les différents ouvrages traitant du sujet (traités, grammaires, dictionnaires). Au XVII ${ }^{\mathrm{e}}$ siècle, A. Furetière inaugure ainsi une définition de la ponctuation par une expression significative :

Observation grammaticale des lieux d'un discours où on doit faire de différentes pauses, \& qu'on marque avec des points $\&$ petits caractères pour en advertir les lecteurs. (Furetière [1690]1970)

Les lieux du discours dans lesquels les signes de ponctuation interviennent se déterminent bien à partir d'une "observation grammaticale", soit la prise en compte essentielle de la syntaxe et du sens. La notion de "pause", souvent invoquée, et considérée généralement comme un critère purement prosodique, doit selon nous se comprendre comme un terme polysémique, dont le spectre sémantique englobe les idées grammaticales de segmentation et de structuration ${ }^{4}$.

3. Selon la lecture qu'effectue É. Benveniste de la théorie saussurienne, le signe est entendu comme unité du système sémiotique de la langue. La sémiotisation, telle que nous l'entendons ici, permet alors de décrire la reconfiguration de l'élément ponctuant en tant que signe (Benveniste [1966]2001: 43).

4. Voir à ce sujet J. Rault " De la "pause" à la "valeur en langue" : grammaticalisation des signes de ponctuation ?", IV ème Congrès mondial de Linguistique française, université libre de Berlin, juillet 2014. 
$\mathrm{Au}$ XVIII ${ }^{e}$ siècle, la réflexion syntaxique sur la phrase et le point (Seguin 1972) contribue à fixer les usages de la ponctuation et participe à l'émancipation de l'approche prosodique, liée à la période. La "grammaticalisation de la notion de phrase » (Seguin 1999 : 8) a entrainé une approche grammaticale des signes de ponctuation dans les discours autorisés. Cette approche s'accompagne d'une entreprise de codification, qui cherche à établir des règles précises : la question de la norme se pose dès lors avec davantage d'acuité. Le Traité pratique de la ponctuation de S.-A. Tassis, par exemple, s'en prend frontalement aux grammairiens et se présente comme un ouvrage destiné à pallier les déficiences de ces derniers, en exposant « les véritables règles de la ponctuation, règles puisées dans la logique [...]». Si le XVIII ${ }^{e}$ siècle a pu développer une réflexion riche sur la ponctuation au point de vue grammatical, le XIX ${ }^{e}$ siècle "s'ingénia davantage, en accord avec lui-même, à la codifier - la manie grammaticalisante des ponctuateurs de l'époque en témoigne - pour la transformer en un élément dont le caractère éternel, qui plus est universel, avait quelque chose de rassurant et de profondément gratifiant " (Demanuelli 1987 : 7). Les enjeux linguistiques d'une telle évolution, on le voit, entrent en résonance avec des enjeux d'ordre anthropologique.

\section{Grammaticalisation}

La morphogénèse de la ponctuation est d'abord l'objet d'un processus qui s'apparente à celui de la grammaticalisation : « la manie grammaticalisante des ponctuateurs " des XVIII ${ }^{\mathrm{e}}$ et XIX ${ }^{\mathrm{e}}$ siècles correspond à une tendance normative qui est l'un des facteurs déclenchants de la grammaticalisation. La motivation est avant tout pragmatique et se traduit par une perte de libertés : les usages tendant à se fixer, jusqu’à devenir « tabou »(Dürrenmatt 2004 : 34). Comme l'a parfaitement bien montré J. Dürrenmatt (Ibid.), l'emploi de la virgule entre le sujet et le verbe devient beaucoup plus difficile à justifier lorsque ce sont les critères syntaxiques et sémantiques qui règlent les usages (sauf dans quelques rares exposés qui prônent la logique contre le grammatical). La grammaticalisation articule ainsi deux versants, morphosyntaxique et sémantique.

Envisager une grammaticalisation des signes de ponctuation impose évidemment de ne pas s'en tenir à une définition restreinte (passage d'une forme au statut lexical à une forme au statut grammatical), mais plutôt de repérer un ensemble de mécanismes et d'évaluer leur pertinence dans ce domaine spécifique et, sans doute, peu compatible (statut catégoriel, autonomie, etc.). Dans un article sur les usages du comma dans un manuscrit du XIII siècle, C. Marchello-Nizia (2008 : 293-306) s'interroge déjà sur l'évolution des fonctions et soumet celle-ci à la notion de grammaticalisation : le signe est-il encore un marqueur d'intonation ? Est-il devenu un marqueur de corrélation entre deux éléments d'un même énoncé ? Cette interrogation sur la grammaticalisation de la virgule à une époque donnée indique qu'une 
telle hypothèse est envisageable pour l'ensemble des signes de ponctuation, à fortiori sur un empan beaucoup plus large.

Les discours autorisés cherchent à conférer au signe de ponctuation un ensemble de fonctions grammaticales. Cette tendance semble avoir permis, autant que rendu nécessaire, une réflexion sur la valeur en langue.

\section{De la grammaire à la langue}

\section{Valeur en langue et interprétations discursives}

De l'élément pneumatique, suprasegmental, indiquant le temps de repos pour la respiration, à l'élément grammatical, syntaxiquement et sémantiquement codé, on constate un net infléchissement, dans le sens d'une restriction de la variété des usages et des fonctions. Cependant, l'absence de départ entre discours et langue, entre usages et valeur, entrave l'entreprise définitoire.

Les descriptions des dictionnaires, des traités mais aussi des grammaires, à dominante fonctionnelle, cherchent le plus souvent à recenser un nombre limité d'usages en discours; dans un souci d'exhaustivité, qui peut trahir un projet normatif, les analyses procèdent par adjonction de fonctions diverses relevant fréquemment de plusieurs niveaux d'analyse (syntaxique, sémantique jusqu'aux interprétations psychologisantes). Une telle entreprise aboutit alors à de longues listes plus ou moins efficientes, l'exemple le plus probant étant sans doute l'inventaire établi par J. Drillon dans son célèbre traité, attribuant notamment au point de suspension pas moins de vingt-six fonctions (1991 : 406-426).

De telles approches définitoires, fondées sur la recension des inépuisables interprétations en discours, relèvent d'une aporie. Faute d'une inscription en langue, les descriptions linguistiques témoignent de la volonté mais aussi de l'impossibilité de définir précisément ces signes singuliers. En suivant les propositions d'Y. Fonagy (1985) et de J. Authier-Revuz (1995, 1998), il semble donc fécond d'appréhender le signe de ponctuation comme un véritable signe linguistique, doté d'un signifiant (graphique et sans correspondant phonémique), et d'un signifié, stable et unique, soit une valeur en langue à partir de laquelle se réaliserait l'infinité des interprétations discursives. Le ponctème, « unité à deux faces, constituée par le signe matériel et sa fonction " (Catach $1980: 21$ ), peut ainsi être transposé du discours à la langue, la "valeur » se substituant à la " fonction ».

Dans cette perspective, J. Authier-Revuz propose de comprendre l'ensemble des usages du guillemet (emprunt à un extérieur discursif, équivoque, adéquation problématique ou pleine adéquation du mot à la chose) à partir de la valeur de "dédoublement opacifiant du dire d'un élément "; le guillemet devient alors une "archi-forme de modalisation autonymique ", instrument " de la simple représentation du fait de dire ce mot-là " (1998: 377-380) ; cette approche, fondatrice, a été suivie de plusieurs travaux, tels 
ceux de S. Pétillon (2002) sur la parenthèse et le tiret ou ceux de J. Lefebvre (2007) sur « l'appel-renvoi de note».

\section{Sémiotisation}

L'évolution des différents discours sur la ponctuation montre l'existence d'un mouvement qui tend vers l'intégration de ces signes dans le système de la langue. On peut alors supposer que le point de vue contemporain s'appuie sur ce qui était à l'œuvre, de façon implicite, dans les codifications grammaticales antérieures. Il fallait bien que le ponctuant, élément marginal, tributaire de l'oral, se grammaticalise pour être ensuite envisagé sur le plan sémiotique : le terme sémiotisation permet ainsi de désigner cette possible reconfiguration du signe de ponctuation dans le champ des unités du système sémiotique de la langue.

La spécialisation sémantique - plutôt qu'un affaiblissement ou qu'une décoloration - sur laquelle repose la grammaticalisation implique la formulation, plus ou moins consciente, d'une valeur fondamentale et la sélection d'un trait spécifique. Le signifié du signe de ponctuation, tel qu'il faudrait l'envisager, serait alors l'explicitation de cette valeur et de ce trait. Le processus de sémiotisation prolonge tout en l'intégrant celui de grammaticalisation, ce qui peut poser la question de la réversibilité puisque l'élément ne redevient pas le signe qu'il était après avoir perdu son statut.

Si la question de l'unidirectionnalité n'est pas vraiment pertinente concernant la seule grammaticalisation, en revanche celle de la réversibilité du processus l'est tout à fait (à condition qu'elle ne soit pas conçue comme un strict retour à la forme d'origine, qui a peu de chances de se produire, en particulier si sont intervenus des phénomènes de réduction phonétique) : il est en effet tout à fait concevable que, une fois grammaticalisée, une forme entame un mouvement inverse, vers un statut moins - ou pas grammatical. Phénomène certes moins fréquent que la grammaticalisation, ce type d'évolution existe cependant bel et bien, comme en témoignent les classiques exemples brandis - à tort selon nous - comme contre-exemples à l'unidirectionnalité de la grammaticalisation. (Prévost 2006 : en ligne)

La grammaticalisation n'est pas un processus irréversible. Et le mouvement qui va de l'encodage grammatical au statut sémiotique est d'autant plus envisageable que l'élément grammaticalisé, suivant un cheminement inverse, n'était pas considéré comme un signe linguistique auparavant.

Certains usages contemporains, dans le discours littéraire, mais aussi médiatique, publicitaire, montrent par exemple un affaiblissement du pouvoir clôturant du point, lequel perd grammaticalement son caractère conclusif. Cette remise en cause de la structure phrastique, s'affranchissant de l'encodage grammatical, pourrait être l'illustration dans le discours d'un cheminement vers un statut moins grammatical, ou " dégrammaticalisé ", qui attesterait, dans l'appréhension intuitive du scripteur cette fois, de 
nouvelles potentialités et pourrait se comprendre à l'aune de la sémiotisation sensible dans les discours spécialisés.

Considérant le processus de grammaticalisation comme un phénomène plus large, ouvert sur trois dimensions (grammaire, syntaxe, langue), c'està-dire comme une étape ayant permis la réflexion sur la langue et le signe, on peut supposer qu'il existe, pour ce champ spécifique des signes de ponctuation, un mouvement de transformation global. Puisque la grammaticalisation suppose une réflexion sur la valeur de base qui a permis l'encodage, sur l'émergence d'un trait de cette valeur, un élément qui n'était pas un signe, une fois codé grammaticalement, peut acquérir le statut de signe linguistique et être intégré dans le système de la langue ; se grammaticalisant, l'élément exhibe son potentiel, c'est-à-dire la possibilité d'existence d'une valeur fondamentale et première : et cela ouvre la voie à une reconfiguration en tant que signe.

\section{Du mot à la phrase}

\section{Définition et terminologie}

Ce nouveau statut impose de reconsidérer la place de ces éléments dans le système, selon la répartition en niveaux. En interrogeant le lieu d'inscription du ponctème dans ses descriptions et dans ses interprétations discursives, on constate qu'il est souvent associé à différents niveaux (du graphème à la page, en passant par le morphème et le mot), faisant office de substitut. Porter la réflexion en langue impose de s'interroger sur la place réelle de la ponctuation dans ce système stratifié.

L'embarras des analystes à situer le signe de langue écrite se ressent autant dans la variation de la terminologie - signes de ponctuation, ponctème, "graphème ponctuo-typographique" (Anis $1988: 246$ ), "ponctogrammes de langue écrite" (Mazziotta 2011:21) - que dans le caractère apophatique des définitions; il suffit de se reporter à la définition (par ailleurs opérante) de C. Tournier $(1977,1980)$ pour s'en rendre compte : non-alphabétique, sans correspondant phonémique (il ne se prononce pas), non-décomposable en unités de rang inférieur. La pluralité des noms métalinguistiques et la récurrence de l'approche en négatif sont révélatrices d'une difficulté majeure liée aux spécificités de ces signes écrits.

Entre la définition linguistique du signe, qui circonscrit par la négative le champ d'action, et les approches syntaxique, sémantique ou encore énonciative qui révèlent un certain nombre de fonctions importantes et parfaitement positives, une contradiction demeure. La plus importante de ces pierres d'achoppement reste la propriété sémantique - cette « tête de Méduse " qui, comme le dit É. Benveniste, est "toujours là, au centre de la langue" (1966: 126) - puisque force est de reconnaitre que les signes de ponctuation sont des éléments essentiels de la constitution du 
discours, porteurs d'une signification et de fonctions, ce qu'une définition exclusivement apophatique tend à négliger.

\section{Plérème}

Contrairement au mot, le signe de ponctuation ne se décompose pas ; cette différence de nature, qui s'explique par un caractère non-alphabétique ( idéogrammatique "si l'on veut), peut être évacuée afin de considérer le reste des propriétés. Si la forme ne permet pas la dissociation en constituants inférieurs, la dimension sémantique autorise en revanche le questionnement sur l'intégration dans différents niveaux.

Pour tenter d'appréhender le rôle linguistique de la ponctuation dans le système, il est nécessaire de reprendre, dans un premier temps, la dichotomie traditionnelle entre ponctuation et " mise en page " (Catach $1980: 18-19$ ), entre ponctuation et «scripturation " (Laufer 1986: 75) ou encore entre "signes de ponctuation " et "ponctuation» (Meschonnic $2000: 290$ ), afin d'envisager la place des signes dans un niveau d'analyse. Maintenir une conception élargie de la ponctuation revient en effet à considérer que celle-ci travaille "la langue à tous les niveaux" (Catach 1985: 65-66); nous limiterons donc l'analyse aux signes de ponctuation noire, doté d'un signifiant ponctuel, en écartant de fait tout ce qui relève de la mise en page et de l'ensemble des effets visuels; cette distinction permettra d'envisager la phrase, sur le modèle d'É. Benveniste, comme « limite supérieure " (1966: 125). Si la parenthèse peut, par exemple, encadrer un paragraphe entier ou un texte, nous considérons qu'elle ne se situe pas pour autant à un niveau supérieur, car ce qui prime n'est pas l'espace textuel encadré mais bien le signe lui-même, son lieu et son mode d'insertion : chacun des signifiants de ce signe double intègre, dans un premier temps, le niveau de la phrase.

S'interrogeant sur la place des signes de ponctuation dans un niveau de l'analyse transposé au système graphique, N. Catach choisit de les intégrer au système plérémique (le plérème étant un signe plein, équivalent écrit du morphème), au sein d'une troisième catégorie nommée «système silencieux » qui comporte également quelques signes grammaticaux, lexicaux et les signes-mots $(1985: 66)^{5}$. La ponctuation " au sens large » concerne toutefois les niveaux supérieurs du " plurisystème graphique ", au-dessus des morphogrammes grammaticaux et lexicaux, et intéresse tous les niveaux de la langue.

5. La spécificité des signes de ponctuation nécessite par ailleurs quelques ajustements, notamment au regard des trois fonctions cumulées par les plérèmes : distinctive-participative (rapports de signifiants à signifiants), classificatoire ou catégorielle (qui tient compte de la position des morphèmes écrits sur la chaine syntagmatique et paradigmatique) et sémantique. Seule la première fonction nommée " distinctive-participative" ne semble pas concerner les ponctèmes dans la mesure où elle traite du rapport de signifiant à signifiant au niveau cénémique. Cet aspect, non-évoqué dans la réflexion globale sur "l'écriture et le signe plérémique ", ne semble toutefois pas constituer un obstacle dans l'assimilation du ponctème au plérème. 
En 2004, J. Anis, interrogeant à son tour la question des niveaux, fait redescendre d'un étage les signes de ponctuation en les situant entre le graphème et le morphème ; s'ils sont au-dessus du graphème alphabétique puisque pourvus d'une "valeur sémantique", ils ne constituent pas en revanche une unité aussi significative que le morphème - ne servant qu'à moduler la signification des séquences alphabétiques (Ibid. : 7). Ce positionnement n'admet pas l'existence d'un réel signifié, soit un contenu sémantique propre (privilège des " alphagrammes" pour J. Anis). En suivant l'hypothèse d'un signifié en langue, "l'auxiliarité » des "topogrammes " (Ibid. : 8) devient moins évidente : chaque signe, pris isolément, met en jeu une valeur spécifique.

Une telle propriété permet de renouer, dans le modèle d'É. Benveniste, avec les différentes réalisations de la catégorie intermédiaire : pourvus d'une véritable signification, qui fait d'eux des unités relativement autonomes, les signes de ponctuation sont comparables aux morphèmes (conjoints ou indépendants) mais aussi aux "mots synnomes"(1966:125), qui ne peuvent entrer dans la phrase que joints à d'autres mots. Le signe de ponctuation est bien une unité discrète, assimilable aux unités de première articulation, qui, pour l'essentiel, s'intègre dans l'unité supérieure qu'est la phrase : celle-ci relève certes "d'un autre ordre de notions" (Ibid : 123), mais on peut reconnaitre que les ponctèmes ont une fonction intégrative (puisqu'ils sont associés à la montée des signes dans la phrase, qu'ils intègrent les signes dans la phrase écrite) et qu'ils deviennent une partie intégrante de celle-ci. De la lettre à la phrase, les signes de ponctuation sont des éléments tout à fait représentatifs du niveau intermédiaire du système et, investissant l'ensemble des subdivisions, doivent être envisagés comme des unités linguistiques absolument significatives.

\section{Conclusion}

Dans le mouvement global qui a fait entrer les signes de ponctuation dans les grammaires et dans le système de l'écrit, la démarche qui porte la réflexion en langue constitue un nouveau pallier de l'évolution des appréhensions, que l'on peut entendre comme un mouvement de sémiotisation : le ponctuant, élément accessoire, presque anecdotique, souvent tributaire d'une conception pneumatique, s'est progressivement grammaticalisé et peut aujourd'hui être envisagé comme une unité linguistique - du système sémiotique de la langue -, unité à laquelle on doit pouvoir attribuer un véritable signifié permettant sans aucun doute de mieux comprendre les réalisations, mais aussi les enjeux et les représentations linguistiques et littéraires qui l'accompagnent.

Les ponctèmes, signes ou plérèmes (du système silencieux), s’inscrivent dans une catégorie " intermédiaire ", relativement ouverte, de la structuration en niveaux; penser cette intégration impose toutefois de prendre en compte plusieurs paramètres essentiels : les spécificités de ces éléments 
(non-alphabétiques donc non-décomposables); la diversité formelle et fonctionnelle des différents signes (de la virgule relativement monovalente au polyvalent point de suspension) ; les enjeux complexes du vaste spectre de la ponctuation au sens large (de la majuscule au blanc de paragraphe, en passant par l'italique, etc.). Autant d'aspects qui font de la ponctuation un lieu idéal, sans doute, pour penser les propriétés de la langue écrite.

Les signes de ponctuation pourraient sans doute faire l'objet d'un traitement plus conséquent, et surtout plus " grammatical ", dans la formation des enseignants. Sur un plan didactique, il s'agirait de s'affranchir de la traditionnelle référence à l'oral, encore très présente dans les manuels scolaires, pour poser les enjeux, indépendamment des phénomènes suprasegmentaux, en termes sémiotiques, syntaxiques et sémantiques. Ainsi, il serait possible d'appréhender les différents usages réalisés à partir de la valeur en langue du signe, ce qui permettrait d'évacuer l'approche normative ${ }^{6}$ au profit d'une démarche descriptive et explicative (les interprétations en discours de la valeur en langue), au plus près des spécificités de la production et de la compréhension de l'écrit.

\section{Julien RAULT}

\section{Références bibliographiques}

- ANIS, J. (1983). Le signifiant graphique. Langue française, 59, 31-44.

- ANIS, J. (2004). Les linguistes français et la ponctuation. L'Information grammaticale, 102, 5-10.

- ANIS, J. (avec J.-L. Chiss \& C. Puech) (1988). L'Écriture : théories et descriptions. Bruxelles : De Boeck-Wesmael.

- AUTHIER-REVUZ, J. (1995). Ces mots qui ne vont pas de soi. Boucles réflexives et non-coïncidences du dire (tome 1). Paris : Larousse, coll. "Sciences du langage ".

- AUTHIER-REVUZ, J. (1998). Le guillemet, un signe de "langue écrite " à part entière. In J.-M. Defays, L. Rosier \& F. Tilkin (dir.), À qui appartient la ponctuation? (pp. 373-388). Bruxelles : Duculot, coll. "Champs linguistiques».

- BENVENISTE, É. ([1966] 2001). Problèmes de linguistique générale (tome 1). Paris : Gallimard, coll. «Tel».

- CATACH, N. (1980). La ponctuation. Langue française, 45, 16-27.

- CATACH, N. (1982). L'écriture et le signe plérémique. Modèles linguistiques, 7 (2), 53-71.

- CATACH, N. (1994). La Ponctuation. Paris : Presses universitaires de France, coll. "Que sais-je ?".

- DEMANUELLI, C. (1987). Points de repère. Centre interdisciplinaire d'études et de recherches sur l'expression contemporaine, université de Saint-Etienne, Travaux LVIII.

6. Dans les programmes des premiers cycles, la ponctuation, très peu présente, est souvent évoquée en dernier lieu, dans une proposition l'associant généralement à l'orthographe : il s'agit de respecter les contraintes syntaxiques, orthographiques, ainsi que la ponctuation. 
- DRILLON, J. (1991). Traité de la ponctuation française. Paris : Gallimard, coll. «Tel».

- DÜRRENMATT, J. (2004). La virgule entre sujet et verbe : petite histoire d'un emploi oublié. L'Information grammaticale, 102, 31-34.

- FONAGY, I. (1980). Structure sémantique des signes de ponctuation. Bulletin de la société de linguistique de Paris, 75, 95-129.

- FURETIÈRE, A. ([1690] 1970). Dictionnaire universel contenant généralement tous les mots français tant vieux que modernes et les termes de toutes les sciences et les arts (tome 3). Paris : Slatkine Reprints.

- LAUFER, R. (1986). L'énonciation typographique: hier et demain. Communication et langages, 68, 68-85.

- LEFEBVRE, J. (2007). La Note comme greffe typographique : étude linguistique et discursive. Thèse de doctorat non-publiée, Sciences du langage, sous la direction de J. Authier-Revuz.

- MARCHELLO-NIZIA, C. (2008). Le comma dans un manuscrit en prose du $13^{\mathrm{e}}$ siècle. Grammaticalisation d'un marqueur de corrélation, ou marquage d'intonation ? In O. Bertrand, S. Prévost, M. Charolles, J. François \& C. Schnedecker (dir.), Discours, diachronie, stylistique du français. Études en hommage à Bernard Combettes (pp. 293-306). Berne : Peter Lang, coll. "Sciences pour la communication »,.

- MAZZIOTTA, N. (2011). Qu'est-ce que la ponctuation ? Définir son objet d'étude dans le cadre d'une recherche des rapports entre ponctuation et syntaxe dans la langue française médiévale. La Ponctuation à la Renaissance (pp. 13-21). Paris : Garnier, coll. «Classique ».

- MESCHONNIC, H. (2000). La ponctuation, graphie du temps et de la voix. La Licorne, 52, 289-293.

- PÉTILLON, S. (2002). Les Détours de la langue. Étude sur la parenthèse et le tiret double. Paris, Louvain: Peeters, coll. "Bibliothèque de l'Information grammaticale ".

- PRÉVOST, S. (2006). Grammaticalisation, lexicalisation et dégrammaticalisation : des relations complexes. Cahiers de Praxématique, 46 http://praxematique. revues.org/638\# bodyftn4>.

- RAULT, J., (2014). De la " pause » à la «valeur en langue » : grammaticalisation des signes de ponctuation ? IV Ème Congrès mondial de Linguistique française, université libre de Berlin, juillet 2014.

- SÉGUIN, J.-P. (1972). La Langue française au XVIII siècle. Paris : Bordas, coll. "Études".

- SÉGUIN, J.-P. (1999). Éléments pour une stylistique de la phrase dans la langue littéraire du XVIII ${ }^{e}$ siècle. L'Information grammaticale, 82, 5-15.

- TASSIS, S.-A. (1859). Traité pratique de la ponctuation. Paris : Librairie de Firmin Didot frères fils \& cie, Imprimeurs de l'institut de France.

- TOURNIER, C. (1977). Pour une approche linguistique de la ponctuation. In N. Catach \& C. Tournier (dir.), La Ponctuation : recherches historiques et actuelles, vol. 2 (pp. 223-243). Paris, Besançon : CNRS \& Groupement de recherches sur les textes modernes.

- TOURNIER, C. (1980). Histoire des idées sur la ponctuation, de l'imprimerie à nos jours. Langue française, 45, 28-40.

- VÉDÉNINA, L.-G. (1989). Pertinence linguistique de la présentation typographique.

Paris, Louvain : Peeters \& Selaf. 